\title{
SFR6 PROTEIN OF FREEZING TOLERANCE IN ARABIDOPSIS DOES NOT AFFECT LOCALIZATION OF CBF1 PROTEIN
}

\author{
Deepthi Lakmini Wathugala*1, Heather Knight ${ }^{2}$ and Marc R. Knight ${ }^{2}$ \\ ${ }^{1}$ Department of Crop Science, Faculty of Agriculture, University of Ruhuna, Sri Lanka \\ ${ }^{2}$ School of Biological and Biomedical Sciences, Durham University, United Kingdom
}

Accepted: $27^{\text {th }}$ January 2014

\begin{abstract}
SFR6 (SENSITIVE TO FREEZING6) is one of plant mediator proteins which has been identified first with its involvement to tolerance against freezing in Arabidopsis. The freezing sensitive sfr6 mutant is lack of expression of downstream genes in CBF cold response pathway. SFR6 also mediates tolerance to osmotic stress induced by drought and salinity. However, sfr6-1 mutant shows normal levels of $C B F$ gene expression suggesting that SFR6 acts downstream of $C B F$ transcription. The over-expression of $C B F 1$ and $C B F 2$ in the sfr6-1 mutant did not increase downstream cold gene expression. Further, SFR6 does not affect the level of accumulation of CBF protein suggesting that SFR6 may effect on localization of CBF proteins in Arabidopsis. Therefore, the localization of CBF1 protein in the sfr6-1 mutant was examined in this study. sfr6-1 and Col-0 plants were crossed with wild type plants over-expressing 35S::CBF1::GFP and F3 generation was used to visualize CBF1 localization. However, the localization of CBF1 protein was not altered in sfr6-1 mutant compared to the wild type Arabidopsis indicating that the involvement of SFR6 for COR gene expression needs to be further studied.
\end{abstract}

Key words: SFR6, Freezing tolerance, Mediator protein

\section{INTRODUCTION}

Developing stress tolerant crops to cope with the rapid environmental degradation that is occurring is an absolute requirement in order to provide enough food for growing population. One of the basic genetic engineering approaches currently being used to improve crop stress tolerance is generation of transgenic plants by introducing novel genes into the genome of agriculturally important crops or altering the expression of existing genes. Understanding stress response signaling pathways is the prime requirement to manipulate stress tolerance of crops by this approach.

The sfr6 mutant of Arabidopsis was isolated on the basis of its failure to cold acclimate (Warren et al., 1996). Further studies showed the freezing sensitivity of the sfr6 mutant is due to greatly reduced levels of the expression of $C O R$ genes controlled by CBF/DREB1 transcription factors in response to low temperature (Knight et al., 1999; Knight et al., 2009). However, CBF/DREB1 gene expression itself was not mis-regulated at the transcriptional and translational level in the sfro- 1 mutant indicating that SFR6 operates downstream of CBF translation (Knight et al., 1999; Knight et al., 2009). In addition sfr6 is compromised in its ability to express genes in response to drought, salinity (Knight et al., 1999; Boyce et al., 2003), UV radiation and pathogen attacks (Wathugala et al., 2012) and in other important developmental processes such as flowering (Knight et al., 2008). At4g04920 locus in chromosome 4 near the centromere was identified as SFR6 locus through mapping and cloning. SFR6 was identified as a $135 \mathrm{kDa}$ protein (Knight et al., 2009) of subunit MED16 of plant mediator complex (Backstrom et al., 2007). Further the over expression of AtSFR6 did not increase KIN2 (downstream gene of CBF pathway) expression in wild type Arabidopsis either under ambient temperatures (Wathugala et al., 2011) or after cold exposure (Wathugala et al., 2012). Thus it is possible that SFR6 controls localization of CBF proteins in Arabidopsis. Therefore, the effect of

\footnotetext{
*Corresponding author: lakmini077@yahoo.com
} 
SFR6 on localization of CBF protein was analyzed in this study.

\section{METHODOLOGY}

Crosses between 35S::AtCBF 1::GFP (GFPGreen Florescent Protein) plants in Col-0 background (kind gift from Prof. M. Thomashow's lab, MSU-DOE plant research lab, Michigan State University) with Col-0 and sfr6-1 mutant plants were performed to construct Arabidopsis lines over-expressing both $C B F 1$ and $G F P$. As $35 S:: A t C B F 1:: G F P$ plants harbour a kanamycin resistant gene F1 plants were selected on kanamycin supplemented MS agar plates. Plants were grown to maturity in a growth room maintained at approximately $20 \pm 1{ }^{\circ} \mathrm{C}$ with a long day photoperiod $(16 \mathrm{~h}$ light $/ 8 \mathrm{~h}$ dark) and light level of 100-150 $\mu \mathrm{mol} \mathrm{m} \mathrm{m}^{-2} \mathrm{~s}^{-1}$ and F2 seeds from individual plants were collected. Seeds were again selected on kanamycin supplemented agar plates and F2 plants were grown for maturity. F3 seeds were grown on kanamycin supplemented agar plates for 12 days. The seeds from Col-0 and sfr6- 1 crossed with $35 S:: A t C B F 1$ were also selected on kanamycin supplemented agar plates. Twelve day old plants were used for segregation of GFP florescence with the sfr6 mutant phenotype. sfr6-1 mutants have pale green colored leaves (Knight et al., 2009). Therefore, seedlings with pale coloured leaves on Kan supplemented plates were selected as homozygous sfr6-1 over-expressing CBF1::GFP. A Confocal laser scanning microscope (Zeiss LSM50) with 40x objectives was used to visualize GFP. The excitation wavelength for GFP visualization was $488 \mathrm{~nm}$, with emission measured using a $505 \mathrm{~nm}$ long pass filter. All images were taken from seedlings grown under similar conditions, or subjected to the same treatments.

\section{RESULTS AND DISCUSSION}

SFR6 gene controls freezing and osmotic stress tolerance in Arabidopsis (Knight et al., 1999, 2009; Boyce et al., 2003). AtSFR6 has been identified as At4g04929 protein (Knight et al., 2009) a plant mediator sub-unit (Backstrom et al., 2007). Overexpression of AtSFR6 in sfr6-1 mutant background complemented all visible phenotypes of sfr6-1 mutant (Wathugala et al., 2011). However, overexpression of AtSFR6 in wild type Arabidopsis did not alter downstream cold gene expression (Wathugala et al., 2011). Further, over-expression of $C B F$ genes in the sfr6-1 mutant did not alter COR gene expression (Knight et al., 2009). However, overexpression of $C B F$ transcription factors in wild type Arabidopsis causes large increase in COR gene expression and freezing tolerance at ambient temperatures (Jaglo-Ottosen et al., 1998; Liu et al., 1998; Kasuga et al., 1999; Gilmour et al., 2000). These results indicate that SFR6 is essential but not sufficient for COR gene expression. Previously, it was also observed that the expression of CBF proteins (translation and protein stability) was not defective in the $s f r 6-1$ mutant (Knight et al., 2009). Therefore, it was suggested that SFR6 might affect CBF activity after CBF translation (Knight et al., 2009). Therefore, it might be possible that SFR6 recruits CBF protein to the nucleus. Therefore, the localization of CBF1 was analyzed by producing wild type and SFR6 plants overexpressing 35S:CBF1:GFP. However, as shown in figure 1 the localization of CBF1 protein was not altered in sfr6-1 mutant compared to the wild type Arabidopsis suggesting that SFR6 is not involved in localization of CBF1 in Arabidopsis.

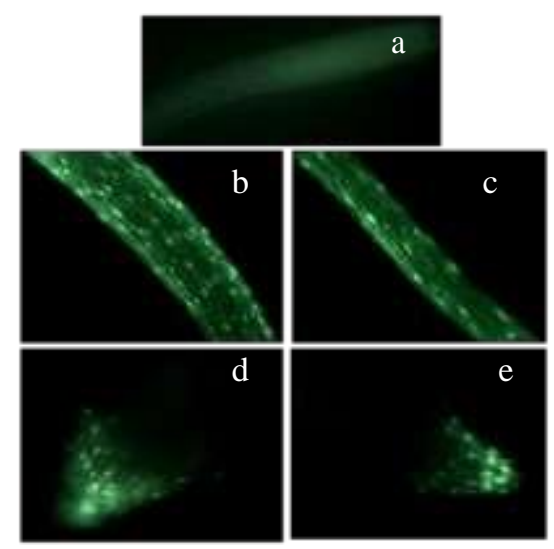

Figure 1: Localization of 35S::CBF1::GFP in root cells of Arabidopsis plants over-expressing 35S::CBF1:GFP. Images were captured using a $40 \times$ objective. (a) Root of wild type Arabidopsis plant over-expressing 35S::CBF1. (b, c) Images of Col-0 and sfr6-1 over-expressing $C B F 1$ and $G F P$ respectively were taken from root elongation zone and (d, e) root cap. Images were taken with identical parameters to allow comparison. 
Mediator is a transcriptional co-activator complex which acts as a bridge to convey DNA-bound transcriptional regulators and enhancers to the general RNA polymerase II transcription machinery (Chadick and Asturias, 2005; Bourbon, 2008). Components of mediator were first identified thought biochemical and genetic studies in baker's yeast (Saccharomyces cerevisiae), as subunits linking to the RNA Polymerase II holoenzyme (Flanagan et al, 1991). Mediator complexes were subsequently found in other organisms including higher eukaryotes (Bourbon et al., 2004; Bjorklund and Gustafsson, 2005; Bourbon, 2008). Of these, the structure and composition of yeast mediator has been studied in most detail. The yeast complex comprises 26 subunits (Guglielmi et al., 2004), and has been grouped through interactor studies into 4 sub-modules known as the head, middle, tail and kinase (CDK) (Asturias et al., 1999). In mammalian mediator, 30 subunits have been identified (Bourbon et al., 2004). However, prior to 2007 there were no reports of a plant mediator complex, probably due to the low sequence homology between Arabidopsis and other non-plant eukaryotic mediators (Backstrom et al., 2007).

Physical interactions between mediator subunits were revealed in 1992 by Jiang and Stillman, through research on yeast SIN4 (MED16) subunit mutant strain. A mutation in the SIN4 (MED16) subunit of yeast showed loss of function of other tail subunits and therefore, it was suggested that mediator subunits physically interact with each other (Jiang and Stillman, 1992). Consistent with these results, electron microscopic (EM) studies of holoenzyme complexes purified from mutant yeast lacking MED16, showed that the tail mediator modules (MED15, MED3 and MED2) were absent (Chadick and Asturias, 2005). Therefore they also suggested that the tail portion of mediator formed a subset of physically interacting units. Moreover, the physical association of yeast MED16 and
MED14 (Rgr1) proteins were also apparent with the same spectrum of phenotypes observed in $\sin 4$ and rgrl mutant strains (Jiang et al., 1995). According to these literatures SFR6/MED16 in Arabidopsis may play very complex role in stress gene regulation. Therefore, further studies of role of SFR6/MED16 will give us clear picture on the mechanism of plant stress gene regulation.

\section{CONCLUSION}

We observed that AtSFR6 has no effect in localization of CBF1 gene. Therefore, the mechanism of regulation of cold induced COR gene expression via SFR6/MED16 remains to be further investigated. Not only SFR6/MED16 subunit, the future research on other subunits and of the whole complex of plant mediator will help us to understand the mechanism of stress gene expression.

\section{REFERENCES}

Asturias FJ, Jiang YW, Myers LC, Gustafsson CM, Kornberg RD (1999) Conserved structures of mediator and RNA polymerase II holoenzyme. Science 283: 985-987

Backstrom S, Elfving N, Nilsson R, Wingsle G, Bjorklund S (2007) Purification of a plant mediator from Arabidopsis thaliana identifies PFT1 as the Med25 subunit. Molecular Cell 26: 717-729

Bjorklund S, Gustafsson CM (2005) The yeast Mediator complex and its regulation. Trends in Biochemical Sciences 30: 240244

Bourbon HM (2008) Comparative genomics supports a deep evolutionary origin for the large, four-module transcriptional mediator complex. Nucleic Acids Research 36: 3993-4008

Bourbon HM, Aguilera A, Ansari AZ, Asturias FJ, Berk AJ, Bjorklund S, Blackwell TK, Borggrefe T, Carey M, Carlson M, Conaway JW, Conaway RC, Emmons SW, Fondell JD, Freedman LP, Fukasawa 
T, Gustafsson CM, Han M, He X, Herman PK, Hinnebusch AG, Holmberg S, Holstege FC, Jaehning JA, Kim YJ, Kuras L, Leutz A, Lis JT, Meisterernest M, Naar AM, Nasmyth K, Parvin JD, Ptashne M, Reinberg D, Ronne H, Sadowski I, Sakurai H, Sipiczki M, Sternberg PW, Stillman DJ, Strich R, Struhl K, Svejstrup JQ, Tuck S, Winston F, Roeder RG, Kornberg RD (2004) A unified nomenclature for protein subunits of Mediator complexes linking transcriptional regulators to RNA polymerase II. Molecular Cell 14: 553557

Boyce JM, Knight H, Deyholos M, Openshaw MR, Galbraith DW, Warren G, Knight MR (2003) The sfr6 mutant of Arabidopsis is defective in transcriptional activation via CBF/DREB1 and DREB2 and shows sensitivity to osmotic stress. Plant Journal 34: 395-406

Chadick JZ and Asturias FJ (2005) Structure of eukaryotic Mediator complexes. Trends in Biochemical Sciences 30: 264-271

Flanagan PM, Kelleher RJ, Sayre MH, Tschochner H and Kornberg RD (1991) A mediator required for activation of RNA polymerase 11 transcription in vitro. Nature 350: 436-438

Guglielmi B, van Berkum NL, Klapholz B, Bijma T, Boube M, Boschiero C, Bourbon HM, Holstege FCP, Werner M (2004) A high resolution protein interaction map of the yeast Mediator complex. Nucleic Acids Research 32: 5379-5391

Gilmour SJ, Sebolt AM, Salazar MP, Everard JD, Thomashow MF (2000) Overexpression of the Arabidopsis CBF3 transcriptional activator mimics multiple biochemical changes associated with cold acclimation. Plant Physiology 124: 1854-1865

Jaglo-Ottosen KR, Gilmour SJ, Zarka DG, Schabenberger O, Thomashow MF (1998) Arabidopsis CBF1 overexpression induces COR genes and enhances freezing tolerance. Science 280: 104-106

Jiang YW, Dohrmann PR, Stillman DJ (1995) Genetic and physical interactions between yeast rgr 1 and $\sin 4$ in chromatin organiza- tion and transcriptional regulation. Genetics 140: 47-54

Jiang YW, Stillman DJ (1992) Involvement of the $\sin 4$ global transcriptional regulator in the chromatin structure of saccharomycescerevisiae. Molecular and Cellular Biology 12: 4503-4514

Kasuga M, Liu Q, Miura S, YamaguchiShinozaki K, Shinozaki K (1999) Improving plant drought, salt, and freezing tolerance by gene transfer of a single stressinducible transcription factor. Nature Biotechnology 17: 287-291

Knight H, Mugford SG, Ulker B, Gao DH, Thorlby G, Knight MR (2009) Identification of SFR6, a key component in cold acclimation acting post-translationally on CBF function. Plant Journal 58: 97-108

Knight H, Thomson AJW, McWatters HG (2008) Sensitive to freezing6 integrates cellular and environmental inputs to the plant circadian clock. Plant Physiology 148: 293-303

Knight H, Veale EL, Warren GJ, Knight MR (1999) The sfr6 mutation in Arabidopsis suppresses low-temperature induction of genes dependent on the CRT DRE sequence motif. Plant Cell 11: 875-886

Liu Q, Kasuga M, Sakuma Y, Abe H, Miura S, Yamaguchi-Shinozaki K, Shinozaki K (1998) Two transcription factors, DREB1 and DREB2, with an EREBP/AP2 DNA binding domain separate two cellular signal transduction pathways in drought- and low-temperature-responsive gene expression, respectively, in Arabidopsis. Plant Cell 10: 1391-1406

Warren G, McKown R, Marin A, Teutonico R (1996) Isolation of mutations affecting the development of freezing tolerance in Arabidopsis thaliana (L) Heynh. Plant Physiology 111: 1011-1019

Wathugala DL, Shane A, Richards Knight H, Knight MR (2011) OsSFR6 is a functional rice orthologue of SENSITIVE TO FREEZING-6 and can act as a regulator of COR gene expression, osmotic stress and freezing tolerance in Arabidopsis, New Phytologist. 191(4): 984-995. 
Wathugala DL, Knight H, Knight MR (2012) MED16/SFR6 is necessary but not sufficient for COR gene expression of $\mathrm{CBF}$ pathway. Journal of Tropical Agricultural research 15(2):16 